\title{
OPEN Distinct expression profiles of regulatory RNAs in the response to biocides in Staphylococcus aureus and Enterococcus faecium
}

\author{
Loren Dejoies $^{1,2}$, Killian Le Neindre ${ }^{1,2}$, Sophie Reissier ${ }^{2}$, Brice Felden ${ }^{2}$ \& Vincent Cattoir ${ }^{1,2,3 凶}$
}

The aim of the study was to characterize the antimicrobial activity of clinically-relevant biocides (chlorhexidine digluconate, benzalkonium chloride, PVP-iodine and triclosan) and to determine the sRNA expression profiles under biocide exposure in two major bacterial opportunistic pathogens, Enterococcus faecium and Staphylococcus aureus. In vitro activities were evaluated against $S$. aureus HG003 and E. faecium Aus0004. We determined MIC, MBC, sub-inhibitory concentrations (SIC) and growth curves under SIC conditions. SRNA expression study under SIC exposure of biocides was performed by RT-qPCR on 3 sRNAs expressed in S. aureus (RNAIII, SprD and SprX) and the first 9 sRNAs identified as expressed in $E$. faecium. MICs were higher against $E$. faecium than for $S$. aureus. Growth curves under increasing biocide concentrations highlighted two types of bactericidal activity: "on/off" effect for chlorhexidine, benzalkonium chloride, PVP-iodine and a "concentrationdependent" activity for triclosan. Exposure to biocide SICs led to an alteration of several sRNA expression profiles, mostly repressed. The distinct biocide activity profiles must be evaluated with other compounds and bacterial species to enrich the prediction of resistance risks associated with biocide usage. Biocide exposure induces various sRNA-mediated responses in both $S$. aureus and $E$. faecium, and further investigations are needed to decipher sRNA-driven regulatory networks.

Resistance to antibiotics keeps increasing worldwide and can lead to treatment failures of severe bacterial infections. "ESKAPE" pathogens include healthcare-relevant Gram-positive and Gram-negative bacteria displaying high capacities for acquisition of resistance determinants, and causing numerous multi-drug resistant (MDR) infections worldwide ${ }^{1}$. Staphylococcus aureus and Enterococcus faecium are two Gram-positive bacteria represented in this ESKAPE group and stand as major human nosocomial pathogens ${ }^{2}$. Searching for novel antimicrobial strategies is essential, but must not be the only option to struggle against infections. Indeed, limiting their occurrence, notably hospital-acquired infections can be achievable with appliance of rigorous hygiene practices in healthcare units. To fulfill these objectives, biocides (incl. antiseptics, disinfectants) are massively used at the hospitals ${ }^{3,4}$. They also include preservative agents and are therefore widely used in industries (e.g. food, cosmetics). As a consequence, the bioaccumulation of various biocides in the environment (food, water, soils) has been evidenced $^{5}$, and some of them, such as triclosan, have been detected in human urine and breast milk ${ }^{6,7}$. Nevertheless, the widespread use of biocides based on clinically-evidenced antimicrobial efficacy is in contrast with the lack of knowledge about their molecular mechanisms of action and resistance. Some are predicted to share common targets with antibiotics, such as cell membranes (chlorhexidine, benzalkonium chloride), while halogenbased biocides (PVP-iodine, triclosan) could have a global antimicrobial effect by inducing an oxidative stress in bacterial cell after passive diffusion through cell membrane ${ }^{8,9}$. In a context of global health concern, the exacerbation of emerging antimicrobial resistance after overuse and/or misuse of biocides has been hypothesized ${ }^{10}$. Indeed, evidences for a link between heavy usage of biocides and emergence of antibiotic resistance are currently highlighted, even though the molecular mechanisms are partially elucidated ${ }^{11-16}$. Furthermore, official guidelines in biocide susceptibility testing are still pending, despite recent propositions have led significant advances in the standardization of procedures ${ }^{17,18}$. As a consequence, the high variability of published results related to different procedures contributes to the misunderstanding of cross-/co-resistance mechanisms. Among various

${ }^{1}$ Department of Clinical Microbiology, Rennes University Hospital, Rennes, France. ${ }^{2}$ Inserm UMR_S 1230, Bacterial Regulatory RNAs and Medicine, University of Rennes 1, Rennes, France. ${ }^{3}$ National Reference Center for Antimicrobial Resistance (Lab 'Enterococci'), Rennes, France. ${ }^{\square}$ email: bfelden@univ-rennes1.fr; vincent.cattoir@ chu-rennes.fr 


\begin{tabular}{|c|c|c|c|c|c|c|c|c|}
\hline & \multicolumn{4}{|c|}{ S. aureus HG003 } & \multicolumn{4}{|c|}{ E. faecium Aus0004 } \\
\hline & \multicolumn{2}{|c|}{ CA-MHB medium } & \multicolumn{2}{|c|}{ BHI medium } & \multicolumn{2}{|c|}{ CA-MHB medium } & \multicolumn{2}{|c|}{ BHI medium } \\
\hline & $\begin{array}{l}\mathrm{MIC}_{4 \mathrm{sh}} \\
(\mathrm{mg} / \mathrm{L})\end{array}$ & $\begin{array}{l}\mathrm{MBC}_{24 \mathrm{~h}} \\
(\mathrm{mg} / \mathrm{L})\end{array}$ & $\begin{array}{l}\text { MBC/MIC } \\
\text { ratio }\end{array}$ & $\begin{array}{l}\text { SIC } \\
(\mathrm{mg} / \mathrm{L})\end{array}$ & $\begin{array}{l}\mathrm{MIC}_{48 \mathrm{~h}} \\
(\mathrm{mg} / \mathrm{L})\end{array}$ & $\begin{array}{l}\mathrm{MBC}_{24 \mathrm{~h}} \\
(\mathrm{mg} / \mathrm{L})\end{array}$ & $\begin{array}{l}\text { MBC/MIC } \\
\text { ratio }\end{array}$ & \begin{tabular}{|l|} 
SIC \\
$(\mathbf{m g} / \mathrm{L})$
\end{tabular} \\
\hline Chlorhexidine digluconate & 2 & 4 & 2 & 1 & 4 & 4 & 1 & 0.5 \\
\hline Benzalkonium chloride & 2 & 8 & 4 & 1 & 4 & 8 & 2 & 2 \\
\hline Triclosan & 2 & 8 & 4 & 0.016 & 8 & 16 & 2 & 0.25 \\
\hline PVP-iodine & 2048 & 2048 & 1 & 1024 & 2048 & 2048 & 1 & 1024 \\
\hline
\end{tabular}

Table 1. Antimicrobial activities of chlorhexidine digluconate, benzalkonium chloride, triclosan and PVPiodine on S. aureus HG003 and E. faecium Aus0004. CA-MHB: cation-adjusted Mueller-Hinton broth; BHI: Brain Heart Infusion; $\mathrm{MIC}_{48 \mathrm{~h}}$ : minimal inhibitory concentrations after $48 \mathrm{~h}$ of incubation; $\mathrm{MBC}_{24 \mathrm{~h}}$ : minimal bactericidal concentrations after $24 \mathrm{~h}$ of incubation; SIC: sub-inhibitory concentration.

strategies triggered by bacteria to cope with environmental changes, regulatory RNAs (sRNAs) are key players among intertwined regulatory networks that mediate dynamic adaptations by modulating gene expression ${ }^{19,20}$. Bacterial adaptation mediated by sRNAs has been exclusively studied under antibiotic conditions ${ }^{21,22}$, but we presume that a biocide stress, especially at subinhibitory concentrations (SICs, concentrations below the MIC, which do not affect bacterial growth), could trigger sRNA-regulated metabolic pathways as well. Among Grampositive human pathogens, sRNAs have been studied in $S$. aureus, with an estimated number close to 50 bona fide sRNAs ${ }^{23}$. Among them, three can be qualified as "clinically-relevant": RNAIII, implicated into the quorum sensing, allowing the transition between expression of adhesive and colonization factors ${ }^{24}$, SprX that contributes to glycopeptide resistance by repressing stage V sporulation protein (spoVG) expression ${ }^{25}$, and SprD that regulates expression of an immune evasion protein (Sbi) and is required for infection on animal models ${ }^{26}$. In Enterococcus spp., sRNAs have been first described in Enterococcus faecalis ${ }^{27,28}$. In E. faecium, the first nine sRNAs whose biological functions remain unknown have been recently discovered in our lab ${ }^{29}$.

In this study, we aimed to characterize the antimicrobial activity of four clinically-relevant biocides (chlorhexidine digluconate, benzalkonium chloride, PVP-iodine and triclosan), and to determine the sRNA expression profiles under biocide stress in two major ESKAPE Gram-positive pathogens, E. faecium and S. aureus. For this aim, we developed different approaches to assess the behavior of those bacteria under biocidal exposure.

\section{Results}

Biocide susceptibility testing. MICs of chlorhexidine digluconate and benzalkonium chloride were twofold higher against $E$. faecium than $S$. aureus $(4 \mathrm{mg} / \mathrm{L}$ and $2 \mathrm{mg} / \mathrm{L}$, respectively), while MICs of triclosan were four-fold higher ( $8 \mathrm{mg} / \mathrm{L}$ and $2 \mathrm{mg} / \mathrm{L}$, respectively) (Table 1). By contrast, MICs of PVP-iodine were identical for both species and 1000-fold higher than the other biocides $(2048 \mathrm{mg} / \mathrm{L})$. MBC/MIC ratios of the four tested biocides suggested a bactericidal activity against both $E$. faecium and $S$. aureus (ratio $\leq 4)(\text { Table } 1)^{9}$.

Bacterial growth under gradient concentrations of biocide revealed that SIC of benzalkonium chloride and triclosan were higher for E. faecium than for S. aureus, whereas SIC of chlorhexidine digluconate was higher for S. aureus than for E. faecium. PVP-iodine SICs were identical for both bacteria and were again about 1000 times higher than the three other biocides (Table 1).

Antibacterial activity profiles. SICs of chlorhexidine digluconate, benzalkonium chloride and PVPiodine were reached at $1 / 2 \times$ MIC for $S$. aureus, as well as for E. faecium except with chlorhexidine digluconate (SIC at $1 / 8 \times$ MIC). SICs of triclosan were much lower than MICs at $1 / 32 \times$ and $1 / 128 \times$ MIC for E. faecium and S. aureus, respectively (Fig. 1 and Table 1). This difference between chlorhexidine, benzalkonium chloride and PVP-iodine in one side, and for triclosan on another was also detected after the analysis of the growth curves. Indeed, two profiles of antibacterial activity were highlighted: chlorhexidine digluconate, benzalkonium chloride and PVP-iodine revealed a weak concentration-dependant activity illustrated by an "on/off" effect on bacterial growth between 1 two-fold dilution. Triclosan revealed a strong concentration-dependent activity resulting in the gradual inhibition of bacterial growth while biocide concentrations increased (Fig. 1).

Overall repression of $E$. faecium sRNAs expression under biocide exposure. Expression profiles of sRNA under each biocide condition are presented in Fig. 2A and Table S1. No significant shifts at the transcript level were observed at the ME phase, except for sRNA_0160 under triclosan $(+151 ; P=0.0008)$. During the later stages, a global repressive trend of sRNAs steady state expression levels was observed. At the LE phase, their expression profiles were similar under chlorhexidine, benzalkonium chloride and triclosan exposure, with a significant decrease of expression for four sRNAs (sRNA_0120, sRNA_0160, sRNA_0280 and sRNA_2210). Besides, sRNA_1300 expression was repressed only under chlorhexidine, while sRNA_0030 expression was repressed only under triclosan. At the ES phase, the expression of some sRNAs was significantly decreased under chlorhexidine (4 out of 9) and benzalkonium chloride (8 out of 9), and the expression of all 9 sRNAs was switched off under triclosan, notably with sRNA_0160 and sRNA_0280 practically extinct $(-45,175 ; P<0.0001$ and $-1009 ; P=0.0002$ respectively). Interestingly, no significant shifts were observed under PVP-iodine at none of the three growth stages. 
E. faecium

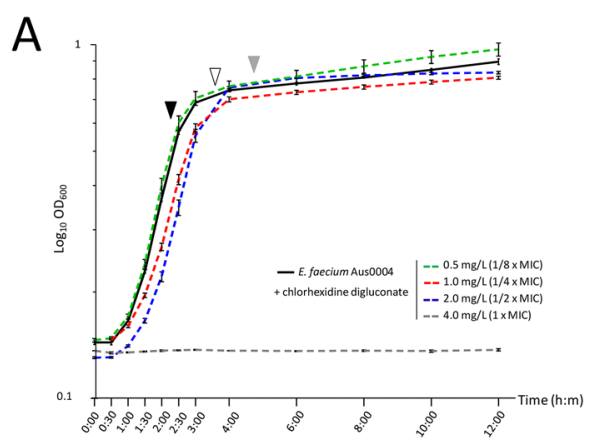

B

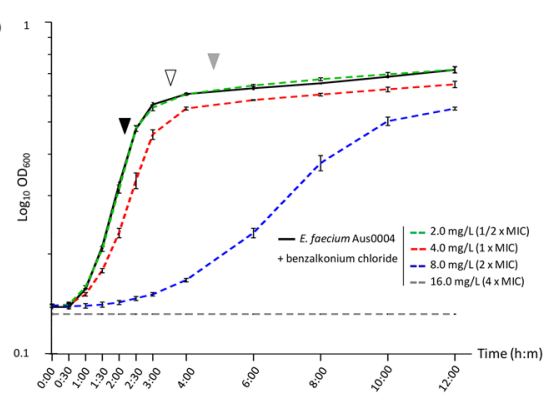

C

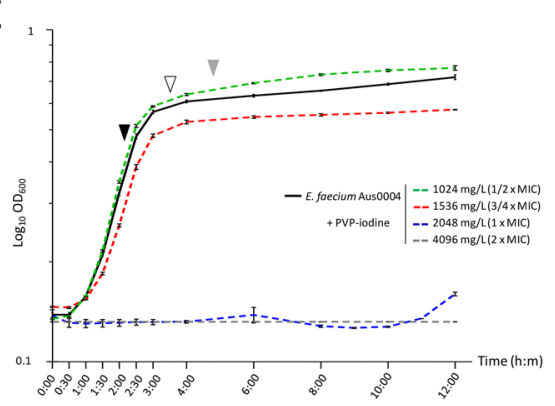

D

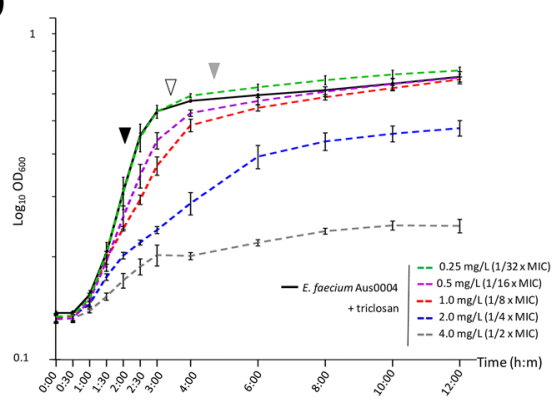

S. aureus
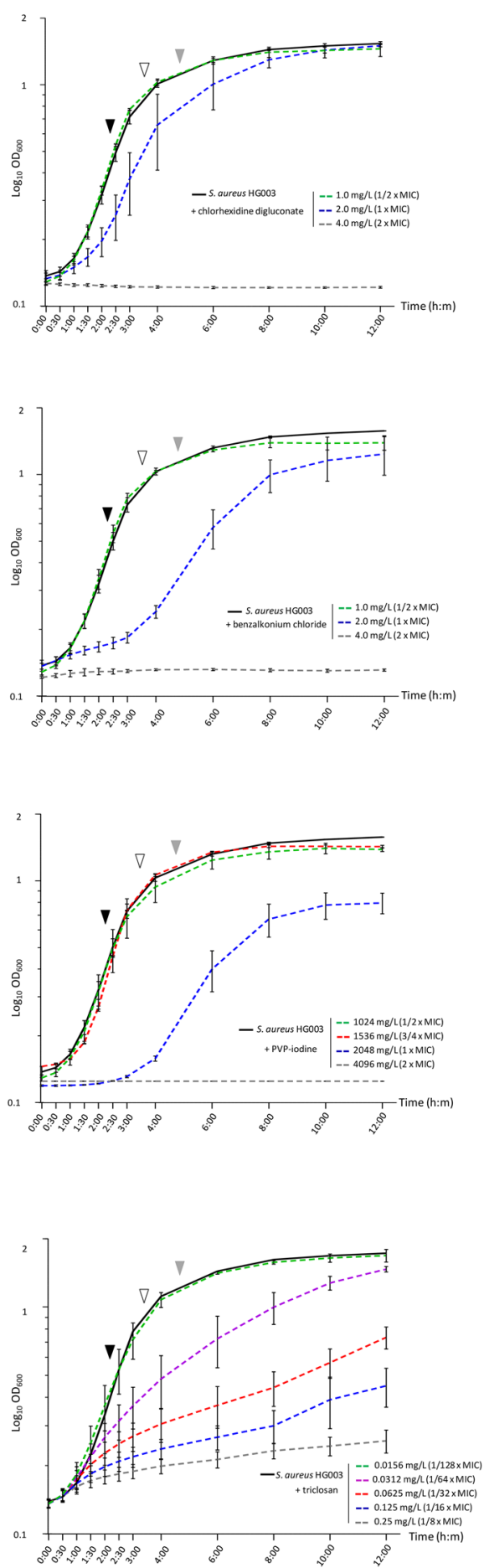

Figure 1. Growth kinetics of E. faecium Aus0004 (lefts panels) and S. aureus HG003 (right panels) under gradients of chlorhexidine (range from 0.5 to $4.0 \mathrm{mg} / \mathrm{L}$ ) (A), benzalkonium chloride (range from 1.0 to $16.0 \mathrm{mg} / \mathrm{L}$ ) (B), PVP-iodine (range from 1024 to $4096 \mathrm{mg} / \mathrm{L}$ ) (C) and triclosan (range from 0.0156 to $4 \mathrm{mg} / \mathrm{L}$ ) (D). Growth curves under sub-inhibitory concentrations (SICs), defined as the highest concentration of biocide for which the standard deviation of the growth curve overlapped with the one of the biocide-free control, are represented in green dashed line. Growth stages considered for sRNA expression corresponded to mid (ME, full black triangle), late (LE, empty black triangle) exponential growth and early stationary (ES, full grey triangle) phases. Error bars indicate standard deviations from three independent experiments. 
A

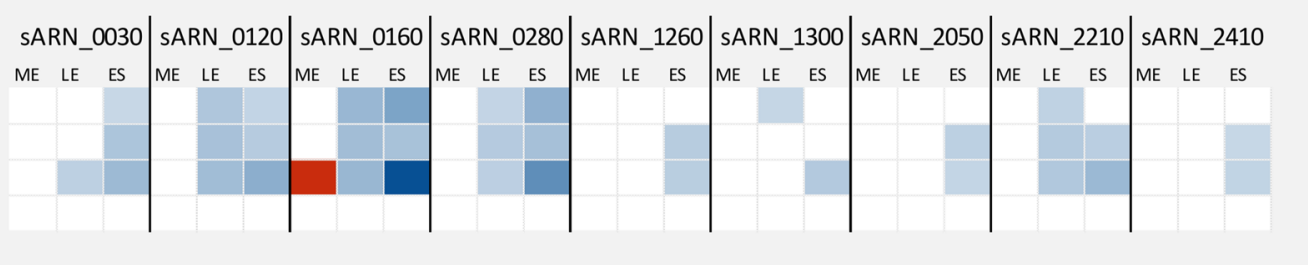

B

Chlorhexidine digluconate Benzalkonium chloride Triclosan

PVP-iodine

(1)

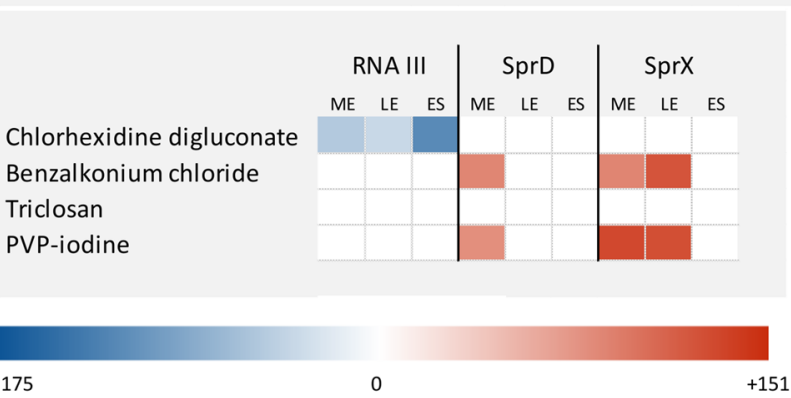

Figure 2. Heat maps illustrating variations in sRNA expression of E. faecium Aus0004 (A) and S. aureus HG003 (B) at mid exponential, late exponential and early stationary growth phases after exposure to SICs of chlorhexidine digluconate, benzalkonium chloride, PVP-iodine and triclosan. RNA counts were normalized against house-keeping genes ( $t m R N A$ for $S$. aureus and adk for E. faecium) using the comparative cycle threshold $\Delta \Delta \mathrm{Ct}$ method and are expressed in $\log _{2}$ fold change. Non-significant shifts in sRNA expression appear in white, significant induction $(P<0.05)$ appears in red, significant repression $(P<0.05)$ are in blue.

Impacts of biocide exposure onto S. aureus sRNAs expression. sRNA expression profiles under each biocide condition are presented on Fig. 2B and Table S2. RNAIII expression profile was significantly influenced $(P<0.05)$ only under chlorhexidine digluconate SIC. Its expression was slightly repressed at ME and LE stages $(-26.8 ; P=0.04$ and $-10.6 ; P=0.0008$ respectively), and mostly at the ES phase $(-1310 ; P=0.04)$. SprX expression profile was altered under two biocides. We observed an increase of expression at the ME and LE stages, then return to normalcy at the DS stage under SICs of benzalkonium chloride $(+18.6 ; P=0.02,+57.9 ; P=0.04$, $+1.6 ; P>0.05)$ and PVP-iodine $(+77.6 ; P=0.02,+66.2 ; P=0.001,+4.9 ; P>0.05)$. SprD expression showed significant shifts under SICs of benzalkonium chloride $(+18.2 ; P=0.003)$ and PVP-iodine $(+14.7 ; P=0.003)$ only at the ME stage. A triclosan exposure did not lead to significant changes in the expression profiles of RNAIII, SprX and SprD.

\section{Discussion}

As expected, the four biocides tested here possessed a potent antimicrobial efficacy and revealed a bactericidal action against both $E$. faecium and $S$. aureus strains (MBC/MIC ratios $\leq 4)^{30}$. The antimicrobial activity of high biocide concentrations found in commercial products (at least at 100-times the MICs) was not investigated in this study because we assessed the effects of low concentrations, mimicking those detected in the environment after their usual uses and misuses. The higher MIC values of chlorhexidine gluconate, benzalkonium chloride and triclosan measured for E. faecium suggest a reduced susceptibility to biocides as compared to $S$. aureus. These results are consistent with inherent ability of Enterococcus spp. to cope with antimicrobial stress ${ }^{31}$, even if they are unlikely exposed to biocides as part of the gastro-intestinal flora, by contrast with $S$. aureus and its cutaneous tropism. These results showing higher MICs in Enterococcus spp are consistent with other studies ${ }^{18,32}$. MIC values are in similar ranges and about 2- to 4-times higher in E. faecium than in S. aureus for every biocide, except for triclosan for which MIC value in S. aureus determined in our study is 16- to 30-times higher than in other studies $^{18,32}$. This discrepancy reveals how raw values can be hardly comparable from one study to another due to a large variability in protocols and interpretation criteria, in addition to intrinsic differences between the tested strains. Indeed, the detection of non-susceptible isolates can fluctuate depending on methodologies used for data acquisition and interpretation. Also, standard protocols to assess the impacts of biocides on bacterial resistance onset are lacking. These major methodological issues were pointed out in 2009 by the Scientific Committee on Emerging and Newly Identified Health Risks (SCENIHR) which recommended standardized methodologies and international surveillance programs to monitor the emergence of biocide resistance (https://ec.europa.eu/ health/ph_risk/committees/04_scenihr/docs/scenihr_o_021.pdf). From there, increasing scientific investigations have led to practical propositions about standardization of biocide susceptibility testing and epidemiological cut-off assessments ${ }^{17,18}$.

Regretfully, we were unable to analyse the MIC/MBC and the SIC data altogether because the culture media were different between the experiments. Indeed, BHI medium was used to perform the biocide stress characterization experiment under sub-lethal biocide concentration because no exploitable data were collected in CA-MHB medium.

Based on the analysis of growth curves under sub-lethal conditions, we enlightened two distinct profiles of antimicrobial activity, independently from their bacterial targets. Chlorhexidine digluconate and benzalkonium 
chloride, both responsible for bacterial membranes disruption ${ }^{8,9}$, as well as PVP-iodine inducing an oxidative stress $^{8,9}$, displayed a weak concentration-dependent effect mimicking an "on-off" effect. These results strengthen the need of concentrated commercial products in clinical use to ensure an optimal antimicrobial activity for these compounds. It also reinforces the hypothesis of a global antibacterial action of these molecules that potentially target numerous molecular components of bacterial cells simultaneously 9 . Triclosan is the only biocide included in our study that displayed a strong concentration-dependent activity. Noteworthy, it is also the only biocide of our panel for which a molecular target is known. Triclosan inhibits lipid synthesis by targeting the enoyl reductase, an essential enzyme involved in the fatty acids elongation cycle, demonstrated in Escherichia coli (FabI enzyme) and in Mycobacterium smegmatis (InhA enzyme) ${ }^{11}$. We hypothesize that triclosan acts similarly in both S. aureus and E. faecium as an antibiotic analog by binding to specific molecular target(s) in agreement with its antibacterial effect that is strongly concentration-dependent.

In this report, we investigated, for the first time, if biocides could influence sRNA expression in two bacterial pathogens, reasoning that biocide are environmental stresses that could be sensed and detected by sRNA-associated gene regulatory networks, to cope with the trigger. sRNA expression study on both S. aureus ( 3 sRNAs), and E. faecium ( 9 sRNAs) in the presence/absence of biocide SIC, revealed that expression levels of several sRNAs is strongly reduced as a result of biocide SIC exposure, although the roles of and the mechanisms connecting those RNAs with the response of bacteria to biocide is unknown. The sRNAs from S. aureus were selected among dozens of expressed sRNA in this bacterium (http://srd.genouest.org/) $/ 3$ because of their implications in either bacterial virulence (SprD , RNAIII) or antibiotic resistance $(\mathrm{SprX})^{24-26}$. Under biocides conditions, RNAIII and SprX were significantly under- and over-expressed depending of the growth stages. Owing to the large number of regulatory functions of RNAIII ${ }^{24}$, its under-expression under chlorhexidine exposure could globally alter the quorum sensing network since RNAIII is the effector. SprX transcript levels followed a similar kinetic under benzalkonium chloride and PVP-iodine, with initial over-expression before returning to normal at the stationary phase. Thus, SprX is implicated into the response towards several antimicrobials targeting cell wall, such as glycopeptides ${ }^{25}$, benzalkonium chloride (this study) or intracellular components as PVP-iodine (this study). The biological functions of sRNAs recently described as expressed in E. faecium ${ }^{29}$ are still under investigations but they could possibly be implicated in gene regulatory networks after a biocide stress is applied and sensed. Among the nine studied sRNAs, sRNA_0160 is a leading candidate due to its substantial repression under chlorhexidine digluconate, benzalkonium chloride, and mostly under triclosan. Furthermore, a previous study showed that sRNA_0160 expression level was significantly downregulated under SIC exposure of daptomycin, an antibiotic targeting the cell wall ${ }^{29}$. Taken together, it suggests that this sRNA would be connected to both the antibiotic and biocide responses in E. faecium, and a deeper understanding of its mechanisms of action would benefit to both fields.

Based on these results, we noticed that sRNA-mediated responses after exposure to four different biocides are distinct between E. faecium and S. aureus. Indeed, triclosan exposure did not lead to significant alteration of sRNAs expression in $S$. aureus, although it was related to an overall repression of sRNAs expression in $E$. faecium. By contrast, PVP-iodine modified SprX expression in S. aureus, but did not influence sRNA expression levels in E. faecium. These results show that biocide exposure induces different sRNA-mediated responses for optimal fitness between the bacterial species. Noteworthy, part of the difference in the transcriptomic response observed between the two strains could be related to their different genetic backgrounds. Indeed, E. faecium Aus0004 is a multi-drug resistant isolate ${ }^{34}$ whereas S. aureus HG003 is a methicillin-susceptible strain ${ }^{35}$ so that we could hypothesize that the adaptation steps necessary for E. faecium to become resistant to antimicrobials could lead to a cross- or a co-resistance selection to biocides. In addition, the expression of a given sRNA can be unchanged, repressed or increased depending on which biocide is used. Furthermore, we should extend our investigations in other bacterial pathogens that may reveal specific variations into their overall sRNA contents under biocides sub-lethal conditions. Likewise, further investigations regarding the functions, mechanisms and molecular targets of these biocide-responding sRNAs are needed to a better understanding of what appear to be complex sRNA-driven regulatory networks allowing biocide adaptation of bacterial pathogens to the presence of biocides into the environment.

\section{Methods}

Bacterial strains. The E. faecium Aus0004 reference strain, isolated from the bloodstream of a patient in Melbourne in 1998, was selected ${ }^{34}$. It is a vancomycin-resistant (van $B$ ) strain belonging to clade A1 that is widely prevalent in healthcare facilities across the world ${ }^{36}$. Its genome was sequenced and annotated in 2003 (Genbank accession no. CP003351). The reference strain of S. aureus was HG003, described as a model strain for physiology and mechanistic studies ${ }^{37}$. It is derived from S. aureus NCTC 8325 (Genbank accession no. NC_007795) that was isolated in 1960 from a septic patient and sequenced in $2001^{35}$.

Biocides. Four clinically-relevant biocides were studied: chlorhexidine digluconate, benzalkonium chloride, PVP-iodine and triclosan (Sigma-Aldrich Corp., St. Louis, MO, USA). Chlorhexidine digluconate, benzalkonium chloride and PVP-iodine stock solutions $(4096 \mathrm{mg} / \mathrm{L})$ were prepared in sterile water and stocked at $4 \pm 2{ }^{\circ} \mathrm{C}$ until use. Triclosan powder, poorly soluble in water, was diluted in ethanol (EtOH) $60 \%$ solvent and freshly prepared at each assay. The suitability of EtOH $60 \%$ solvent was confirmed by innocuity testing (data not shown). Dilutions were prepared from stock solutions in culture media adapted to the experiments: cation-adjusted Mueller-Hinton broth (CA-MHB) (Sigma-Aldrich Corp., St. Louis, MO, USA) for MICs and MBCs assays, brain-heart infusion (BHI) broth (Sigma-Aldrich Corp., St. Louis, MO, USA) for sub-lethal conditions assays, and bacterial cultures for RNA isolation. 
Antimicrobial activity testing. MICs were determined by the broth microdilution (BMD) method in $\mathrm{CA}-\mathrm{MHB}$ medium after 24 and $48 \mathrm{~h}$ incubation at $35 \pm 2{ }^{\circ} \mathrm{C}$. Standardized inocula and step-dilutions of biocides were derived from EUCAST recommendations for antibiotic susceptibility testing ${ }^{38}$. MBCs were determined by sub-culturing on Mueller-Hinton agar plates the wells of MIC plate exhibiting bacterial growth inhibition after $24 \mathrm{~h}$ of incubation and were defined as the lowest concentration of biocide that reduces the initial bacterial inoculum by $\geq 99.9 \%$ (i.e., $-3 \log _{10}$ ). Each assay was performed at least in triplicate.

For biocide stress characterization, bacterial strains were exposed to sub-lethal concentrations of biocides. No bacterial growth was observed in microplates in CA-MHB medium therefore, the experiment was performed in $\mathrm{BHI}$ medium. Standardized bacterial suspensions in BHI medium were exposed to a biocide gradient (from $1 \times$ to $1 / 128 \times \mathrm{MIC})$ at $37^{\circ} \mathrm{C}$ without agitation for $12 \mathrm{~h}$. Optical density at $600 \mathrm{~nm}\left(\mathrm{OD}_{600}\right)$ was measured at 30 -min intervals using a Synergy 2 multi-mode reader (Biotek). The reliability between OD variations and bacterial growth has been assessed by a positive correlation between OD values and CFU/mL in BHI medium without biocide $\left(\mathrm{R}_{\text {Pearson }}^{2}>0.9\right)$ and by the absence of interference of biocide in BHI medium on OD values. Each assay was performed at least in triplicate. Growth curves were represented with the GraphPad Prism software v5.0 (San Diego, CA, USA). Sub-inhibitory concentrations (SIC) corresponded to the highest concentration of biocide for which the standard deviation of the growth curve overlapped with the one of the biocide-free control.

Bacterial Cultures, RNA Isolation, and Expression Analysis. Enterococcus faecium and Staphylococcus aureus strains were grown in BHI medium and then harvested at three different growth stages (midexponential [ME], late exponential [LE] and early stationary [ES]) that were determined at 2:15, 3:30 and 4:45 of growth time respectively. Cells were isolated by centrifugation and dissolved in a solution of $33 \mathrm{mM}$ sodium acetate, $17 \mathrm{mM}$ sodium dodecyl sulfate, and $1 \mathrm{mM}$ EDTA ( $\mathrm{pH}$ 5.5). The cells were then mixed with glass beads and lysed by using a Fast Prep instrument (MP Biochemicals, LLC, Santa Ana, CA, USA).

Total RNAs were isolated by using water-saturated phenol ( $\mathrm{pH}$ 5.0). RNAs were precipitated and washed with ethanol. Depletion of residual DNA was performed using the TURBO DNA-free kit (Invitrogen ${ }^{\text {Tn }}$, Thermo Fisher Scientific). Three independent experiments were performed, with independent RNA purifications.

Expression levels of regulatory RNAs in strains were monitored by using a 2-step quantitative reversetranscription PCR. Briefly, cDNAs were produced by using a High-Capacity cDNA Reverse Transcription Kit (Applied Biosystems) and amplified by using SYBR Green (Power SYBR ${ }^{\mathrm{pm}}$ Green PCR Master Mix, Thermo Fisher Scientific) and specific primers, as previously described ${ }^{25,26,29}$. Regulatory RNA counts were normalized against house-keeping genes ( $t m R N A$ for $S$. aureus, and adk for E. faecium) using the comparative cycle threshold $\Delta \Delta \mathrm{Ct}$ method $^{39}$. Statistical t-test was performed to analyse the $\Delta \mathrm{Ct}$ under biocide condition compared to the control condition, as described ${ }^{40}$. Fold-differences calculated using the $\Delta \Delta \mathrm{CT}$ method were expressed as a range, with incorporation of the standard deviation of the $\Delta \Delta \mathrm{CT}$ value into the fold-difference calculation.

Received: 14 August 2020; Accepted: 5 March 2021

Published online: 25 March 2021

\section{References}

1. Rice, L. B. Federal funding for the study of antimicrobial resistance in nosocomial pathogens: No ESKAPE. J. Infect. Dis. 197, 1079-1081 (2008).

2. Tacconelli, E. et al. Discovery, research, and development of new antibiotics: the WHO priority list of antibiotic-resistant bacteria and tuberculosis. Lancet Infect. Dis. 18, 318-327 (2018).

3. SF2H. Gestion pré-opératoire du risque infectieux. Conférence de consensus 2004 actualisée en 2013. 2013.

4. Fraise, A. P. Choosing disinfectants. J. Hosp. Infect. 43, 255-264 (1999).

5. Gaze, W. H., Abdouslam, N., Hawkey, P. M. \& Wellington, E. M. H. Incidence of class 1 integrons in a quaternary ammonium compound-polluted environment. Antimicrob Agents Chemother 49, 1802-1807 (2005).

6. Dhillon, G. et al. Triclosan: Current Status, Occurrence, environmental risks and bioaccumulation potential. Int. J. Environ. Res. Public Health 12, 5657-5684 (2015).

7. Adolfsson-Erici, M., Pettersson, M., Parkkonen, J. \& Sturve, J. Triclosan, a commonly used bactericide found in human milk and in the aquatic environment in Sweden. Chemosphere 46, 1485-1489 (2002).

8. Fleurette J, Freney J, Everdy ME. Antisepsie et désinfection. ESKA, 1995: 155-168.; 1995.

9. Lorian, V. Antibiotics in laboratory medicine 5th edn. (Lippincott Williams \& Wilkins, 2005).

10. Maillard, J.-Y. et al. Does microbicide use in consumer products promote antimicrobial resistance? A critical review and recommendations for a cohesive approach to risk assessment. Microb. Drug Resist. Larchmt N 19, 344-354 (2013).

11. McMurry, L. M., McDermott, P. F. \& Levy, S. B. Genetic evidence that InhA of Mycobacterium smegmatis is a target for triclosan. Antimicrob. Agents Chemother. 43, 711-713 (1999).

12. Sidhu, M. S., Heir, E., Leegaard, T., Wiger, K. \& Holck, A. Frequency of disinfectant resistance genes and genetic linkage with beta-lactamase transposon Tn552 among clinical staphylococci. Antimicrob. Agents Chemother. 46, 2797-2803 (2002).

13. Chuanchuen, R. et al. Cross-resistance between triclosan and antibiotics in Pseudomonas aeruginosa is mediated by multidrug efflux pumps: exposure of a susceptible mutant strain to triclosan selects nfxB mutants overexpressing MexCD-OprJ. Antimicrob. Agents Chemother. 45, 428-432 (2001).

14. Harkins, C. P. et al. The widespread use of topical antimicrobials enriches for resistance in Staphylococcus aureus isolated from patients with atopic dermatitis. Br. J. Dermatol. 179, 951-958 (2018).

15. Nicolae Dopcea, G., Dopcea, I., Nanu, A., Diguta, C. \& Matei, F. Resistance and cross-resistance in Staphyloccocus sp. after prolonged exposure to different antiseptics. J. Glob. Antimicrob. Resist. 21, 399-404 (2019).

16. Verspecht, T. et al. Development of antiseptic adaptation and cross-adaptation in selected oral pathogens in vitro. Sci. Rep. 9, 8326 (2019).

17. Schug, A. R. et al. Biocide susceptibility testing of bacteria: Development of a broth microdilution method. Vet. Microbiol. 248, 108791 (2020).

18. Morrissey, I. et al. Evaluation of epidemiological cut-off values indicates that biocide resistant subpopulations are uncommon in natural isolates of clinically-relevant microorganisms. PLOS ONE 9, e86669 (2014). 
19. Wagner, E. G. H. \& Romby, P. Small RNAs in bacteria and archaea: who they are, what they do, and how they do it. Adv. Genet. 90, 133-208 (2015).

20. Klein, G. \& Raina, S. Small regulatory bacterial RNAs regulating the envelope stress response. Biochem. Soc. Trans. 45, 417-425 (2017).

21. Lalaouna, D., Eyraud, A., Chabelskaya, S., Felden, B. \& Massé, E. Regulatory RNAs involved in bacterial antibiotic resistance. PLoS Pathog. 10, e1004299 (2014).

22. Howden, B. P. et al. Analysis of thesmall RNA transcriptional response in multidrug-resistant Staphylococcus aureus after antimicrobial exposure. Antimicrob. Agents Chemother. 57, 3864-3874 (2013).

23. Liu, W. et al. Assessment of bona fide sRNAs in Staphylococcus aureus. Front. Microbiol. 9, 288 (2018).

24. Bronesky, D. et al. Staphylococcus aureus RNAIII and its regulon link quorum sensing, stress responses, metabolic adaptation, and regulation of virulence gene expression. Annu. Rev. Microbiol. 70, 299-316 (2016)

25. Eyraud, A., Tattevin, P., Chabelskaya, S. \& Felden, B. A small RNA controls a protein regulator involved in antibiotic resistance in Staphylococcus aureus. Nucleic Acids Res. 42, 4892-4905 (2014).

26. Chabelskaya, S., Gaillot, O. \& Felden, B. A Staphylococcus aureus small RNA is required for bacterial virulence and regulates the expression of an immune-evasion molecule. PLoS Pathog. 6, e1000927 (2010).

27. Shioya, K. et al. Genome-wide identification of small RNAs in the opportunistic pathogen Enterococcus faecalis V583. PLoS ONE 6, e23948 (2011).

28. Michaux, C. et al. Involvement of Enterococcus faecalis small RNAs in stress response and virulence. Infect. Immun. 82, 3599-3611 (2014).

29. Sinel, C. et al. Small RNAs in vancomycin-resistant Enterococcus faecium involved in daptomycin response and resistance. Sci. Rep. 7, 11067 (2017).

30. Pankey, G. A. \& Sabath, L. D. Clinical relevance of bacteriostatic versus bactericidal mechanisms of action in the treatment of Gram-positive bacterial infections. Clin. Infect. Dis. Off. Publ. Infect. Dis. Soc. Am. 38, 864-870 (2004).

31. Guzman Prieto, A. M. et al. Global emergence and dissemination of enterococci as nosocomial pathogens: attack of the clones?. Front. Microbiol. 7, 788 (2016).

32. Koburger, T., Hübner, N.-O., Braun, M., Siebert, J. \& Kramer, A. Standardized comparison of antiseptic efficacy of triclosan, PVPiodine, octenidine dihydrochloride, polyhexanide and chlorhexidine digluconate. J. Antimicrob. Chemother. 65, 1712-1719 (2010).

33. Sassi, M. et al. SRD: a Staphylococcus regulatory RNA database. RNA 21, 1005-1017 (2015).

34. Lam, M. M. C. et al. Comparative analysis of the first complete Enterococcus faecium genome. J. Bacteriol. 194, 2334-2341 (2012).

35. Herbert, S. et al. Repair of global regulators in Staphylococcus aureus 8325 and comparative analysis with other clinical isolates. Infect. Immun. 78, 2877-2889 (2010).

36. Lebreton, F. et al. Emergence of epidemic multidrug-resistant Enterococcus faecium from animal and commensal strains. MBio 4, e00534-13-e00534-13 (2013).

37. Sassi, M., Felden, B. \& Augagneur, Y. Draft genome sequence of Staphylococcus aureus subsp. aureus strain HG003, an NCTC8325 derivative. Genome Announc. 2, e00855-14-e00855-14 (2014).

38. European Committee for Antimicrobial Susceptibility Testing (EUCAST) of the European Society of Clinical Microbiology and Infectious Disease (ESCMID). Determination of minimum inhibitory concentrations (MICs) of antibacterial agents by broth dilution. Clin. Microbiol. Infect. 2003; 9: 1-7.

39. Wong, M. L. \& Medrano, J. F. Real-time PCR for mRNA quantitation. Biotechniques 39, 75-85 (2005).

40. Yuan, J. S., Reed, A., Chen, F. \& Stewart, C. N. Statistical analysis of real-time PCR data. BMC Bioinf. 7, 85 (2006).

\section{Acknowledgements}

S.R. is the recipient of a fellowship funded by the Fondation pour la Recherche Médicale (FRM). This work was funded by the Agence Nationale pour la Recherche (grant number ANR-15-CE12-0003-01 'sRNA-Fit' to B.F.), by the FRM (grant number DBF20160635724 'Bactéries et champignons face aux antibiotiques et antifongiques' to B.F.; personal grant as a member of the scientific council to V.C.) and by the Institut National de la Santé et de la Recherche Médicale (INSERM).

\section{Author contributions}

V.C. and B.F. conceived and designed the experiments; L.D., K.L.N. and S.R. performed the experiments; L.D., K.L.N., S.R., B.F. and V.C. analysed the data; V.C. and B.F. contributed with reagents/materials/analysis tools; L.D., B.F. and V.C. wrote the manuscript. All the authors discussed the results and commented on the manuscript.

\section{Competing interests}

The authors declare no competing interests.

\section{Additional information}

Supplementary Information The online version contains supplementary material available at https://doi.org/ 10.1038/s41598-021-86376-y.

Correspondence and requests for materials should be addressed to B.F. or V.C.

Reprints and permissions information is available at www.nature.com/reprints.

Publisher's note Springer Nature remains neutral with regard to jurisdictional claims in published maps and institutional affiliations. 
(c) (i) Open Access This article is licensed under a Creative Commons Attribution 4.0 International cc) License, which permits use, sharing, adaptation, distribution and reproduction in any medium or format, as long as you give appropriate credit to the original author(s) and the source, provide a link to the Creative Commons licence, and indicate if changes were made. The images or other third party material in this article are included in the article's Creative Commons licence, unless indicated otherwise in a credit line to the material. If material is not included in the article's Creative Commons licence and your intended use is not permitted by statutory regulation or exceeds the permitted use, you will need to obtain permission directly from the copyright holder. To view a copy of this licence, visit http://creativecommons.org/licenses/by/4.0/.

(C) The Author(s) 2021 